\title{
Predictors of Antenatal Insulin Requirement in Women With Gestational Diabetes Mellitus: A Prospective Observational Cohort Study
}

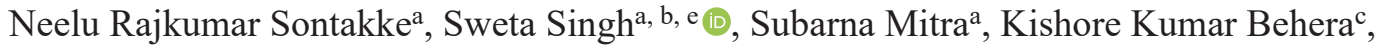 \\ Pankaj Kumar Mohantyd
}

\begin{abstract}
Background: The treatment of gestational diabetes mellitus (GDM) includes medical nutrition therapy (MNT), and insulin if MNT fails to achieve glycemic control. Limited data are available on predictors of insulin requirement in pregnancy and the difference in obstetric outcomes between the two treatment groups. This study was conducted with the primary objective of identifying predictors of antenatal insulin requirement and the secondary objective of comparing perinatal outcomes in MNT-treated versus insulin-treated groups.
\end{abstract}

Methods: It was a prospective cohort study at a tertiary care institute in eastern India. Antenatal women were diagnosed with GDM as per the Diabetes in Pregnancy Study Group of India criteria and received MNT for 1 - 2 weeks, depending on gestational age, followed by self-monitoring of blood glucose. Insulin therapy was added in those who could not meet the target blood glucose. Maternal and neonatal outcomes were studied in these two groups and an attempt was made to identify the predictors of antenatal insulin requirement. The perinatal outcomes in MNT-treated versus insulin-treated groups were also compared.

Results: Data of 151 participants were analyzed. Eighty percent were controlled with MNT. Gestational age $\geq 33.28$ weeks at diagnosis of GDM, maternal BMI $\geq 24.7 \mathrm{~kg} / \mathrm{m}^{2}$ and 75 -gram oral glucose tolerance test (75-g OGTT) value $\geq 162 \mathrm{mg} / \mathrm{dL}$ were predictors of antenatal insulin requirement. The median age, weight, body mass index (BMI), value of 75-g OGTT at diagnosis of GDM, number of cesarean deliveries, gestational hypertension and neonatal hyperbilirubinemia were significantly higher in the insulin group.

Manuscript submitted January 20, 2022, accepted February 4, 2022

Published online February 15, 2022

aDepartment of Obstetrics and Gynecology, All India Institute of Medical Sciences, Bhubaneswar, India

bKing's College Hospital, London, UK,

'Department of Endocrinology, All India Institute of Medical Sciences, Bhubaneswar, India

${ }^{\mathrm{d}}$ Department of Neonatology, All India Institute of Medical Sciences, Bhubaneswar, India

${ }^{e}$ Corresponding Author: Sweta Singh, Department of Obstetrics and Gynaecology, All India Institute of Medical Sciences, Bhubaneswar, Odisha 751019, India. Email: obgyn_sweta@aiimsbhubaneswar.edu.in

doi: https://doi.org/10.14740/jem793
Conclusion: Gestational age at diagnosis of GDM, BMI and value of 75-g OGTT predicted antenatal insulin requirement. Gestational hypertension, rate of cesarean deliveries and neonatal hyperbilirubinemia were significantly higher in the insulin group.

Keywords: Gestational diabetes mellitus; Medical nutrition therapy; Insulin; Predictors

\section{Introduction}

Gestational diabetes mellitus (GDM) is defined as carbohydrate intolerance of variable severity with onset or first recognition during pregnancy [1]. In a worldwide estimate, hyperglycemia in pregnancy was noted in 20 million or $16 \%$ of all live births, of which $84 \%$ or one in six births was estimated to be due to GDM [2]. In Asia, GDM complicates approximately $11.5 \%$ of all pregnancies [3]. Indians have a higher risk for developing GDM, with an estimated prevalence of around $15.5 \%$, depending on the geographical location and diagnostic criteria used $[4,5]$.

GDM is associated with adverse pregnancy outcomes affecting both mother and fetus [6]. Good glycemic control throughout pregnancy can effectively improve adverse fetomaternal outcomes [7-10]. This is achieved initially by medical nutrition therapy (MNT) which includes diet modification, glucose monitoring and moderate exercise. If MNT fails to achieve glycemic control, pharmacological therapy becomes necessary with insulin as the drug of choice. Insulin is usually administered as a subcutaneous injection in two or three divided doses daily.

However, the predictors of insulin requirement in pregnancy are not well described. There is also paucity of data on the difference in perinatal outcomes between women with GDM who achieve glycemic control with MNT versus those in whom insulin-therapy is warranted. This has implications during counselling of women, especially in resource-constrained areas. Hence, this prospective cohort study was conducted with the primary objective to identify the various parameters that predict antenatal insulin requirement. The secondary objective was to compare the maternal and neonatal outcomes in women with GDM treated with MNT versus those treated with addition of insulin. 


\section{Materials and Methods}

\section{Study design and settings}

The study was a prospective observational cohort study conducted in the Department of Obstetrics and Gynaecology of a tertiary teaching hospital in Eastern India, after obtaining clearance from the Institute Ethics Committee (IEC/AIIMS BBSR/PG Thesis/2018-19/24; dated July 06, 2018). The study was conducted in compliance with the ethical standards of the responsible institution on human subjects as well as with the Helsinki Declaration. The study period was 2 years.

\section{Inclusion and exclusion criteria}

All antenatal women diagnosed with gestational diabetes mellitus according to Diabetes in Pregnancy Study Group in India (DIPSI) criteria [11], age between 20 and 35 years and having singleton pregnancy at less than 37 weeks of gestation during the study period were recruited into the study. Those with overt diabetes mellitus, hypertension in pregnancy detected prior to diagnosis of GDM, unwilling to give consent for the study and those on oral hypoglycemic agents were excluded from the study.

\section{Study protocol}

All pregnant women were subjected to 75-gram oral glucose tolerance test (75-g OGTT) at their first antenatal visit as per DIPSI criteria irrespective of the fasting status or last meal [11]. The $75 \mathrm{~g}$ glucose was weighed and dissolved in $200-300$ $\mathrm{mL}$ of water, if required lemon was added for taste. The participants were asked to drink this glucose solution within 5 - 10 min. They were also asked to avoid taking a meal or doing vigorous activity for $2 \mathrm{~h}$, until the blood sample was taken. After $2 \mathrm{~h}$ a venous blood sample was taken, and blood glucose level was measured. If the participant had vomiting within half an hour, the test was repeated the next day. If vomiting occurred after $30 \mathrm{~min}$, the test was continued. A cut-off value of $\geq 140$ $\mathrm{mg} / \mathrm{dL}$ was considered as a positive result and the participant was diagnosed to have GDM and recruited into the study. If the value was normal, the test was repeated between 24 and 28 weeks and again at 32 - 34 weeks.

At recruitment, detailed history including participant's age, parity, obstetric score, gestational age at diagnosis of GDM and the value of 75-g OGTT were recorded. Thorough clinical examination with the measurement of height, weight, body mass index (BMI), blood pressure and obstetric examination was done. The participants diagnosed with GDM were advised MNT for 2 weeks (1 week if $>32$ weeks gestation). MNT was constituted of diet and exercise. Diet was constituted of carbohydrate $50-60 \%$, proteins $20 \%$ and fats $30-40 \%$. The caloric requirement was calculated based on pre-pregnancy ideal body weight. The total calories calculated were divided into nine portions. Every two portions constituted a major meal, and every one portion amounted to a snack. A diet chart was given to each participant in consultation with dietician based on the total calories calculated. Participants did 10 min of exercise (brisk walking or arm exercises) after each major meal constituting $30 \mathrm{~min} /$ day for at least five times a week. Self-monitoring of fasting and 2-h postprandial capillary glucose was done at least twice per week at home using glucometer which was bought by the patient.

All participants were monitored for fetal growth and wellbeing as per institutional protocol. For women well controlled on MNT, induction of labor (IOL) was done at 40 weeks and at 38 weeks for women controlled with insulin, if they did not go to spontaneous labor earlier, as per institute protocol. Labor was also induced for non-GDM-related maternal or fetal indications like gestational hypertension, preeclampsia, pre-labor rupture of membranes, fetal growth restriction, oligohydramnios, etc. Cesarean delivery was performed for obstetric indications only.

\section{Outcome measures}

Maternal outcomes considered were development of gestational hypertension, cesarean delivery, instrumental delivery, postpartum hemorrhage, surgical site infection and uncontrolled blood sugars.

Fetal outcomes considered were large for gestational age (LGA, birth weight $>$ 90th percentile), neonatal hypocalcemia (ionized calcium levels were measured at birth using cord blood, a value $<1 \mathrm{mmol} / \mathrm{L}$ defined hypocalcemia), cardiac anomalies, neonatal hypoglycemia (measured by heel prick, using plasma calibrated glucometer, a value $<45 \mathrm{mg} / \mathrm{dL}$ defined hypoglycemia in a neonate), neonatal polycythemia (at $6 \mathrm{~h}$ of life packed cell volume was measured and value $>65 \%$ was considered abnormal) and neonatal hyperbilirubinemia (all neonates were subjected to trans cutaneous bilirubin (TCB) measurement, the value of which was plotted on a graph for phototherapy by the American Academy of Pediatrics (AAP) and those having value above the cut-off underwent serum bilirubin estimation and phototherapy accordingly).

\section{Statistical analysis}

Data were collected manually for all participants and were entered using Microsoft Excel 2019. Statistical analysis was done using Statistical Package for the Social Sciences (SPSS) version 23. The baseline characteristics were tested for their normalcy using the Shapiro-Wilk test and all variables were found to be not normally distributed. Thus, the data have been summarized as median (interquartile range). For continuous variables, Wilcoxon-Mann-Whitney U test and for categorical data Chi-squared test were used to test the difference between two groups and a $\mathrm{P}$ value of $<0.05$ was considered significant. Descriptive analysis of categorical variables like parity, gestational age at diagnosis, gestational age at the time of delivery, features of severity in hypertensive disorders of pregnancy, and low birth weight was presented as frequency (percentage). Chi-squared test was used to identify the difference between maternal characteristics (age, 


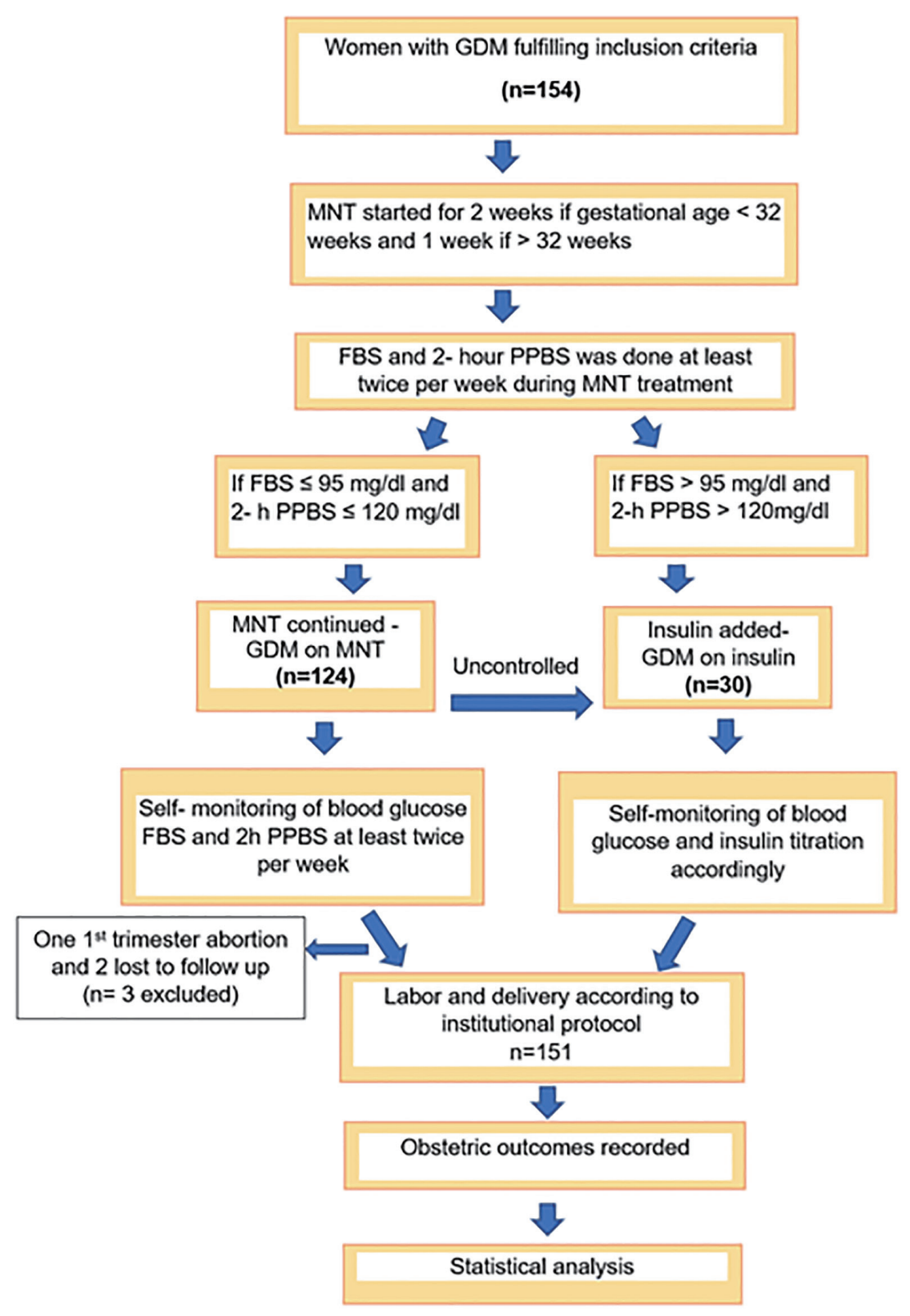

Figure 1. STROBE flow diagram.

parity, gestational age at diagnosis and 75-g OGTT value) in the two groups (MNT and insulin). For a similar analysis of BMI, Fisher's exact test was used as more than $20 \%$ of the total number of cells had an expected count of less than 5 . Receiver operating characteristic (ROC) curve was used in an attempt to establish the cut-off for BMI and value of 75-g OGTT, which would predict and identify GDM participants who would require insulin.

\section{Results}

A total of 154 women were recruited into the study during the study period. One participant had spontaneous first-trimester abortion and two were lost to follow-up. Therefore, a total of 151 participants were analyzed (Fig. 1).

Out of 151 participants, $121(80.1 \%)$ were well controlled on MNT. Insulin was required in 30 (19.9\%) participants. Table 1 depicts the baseline maternal characteristics. The median age of all participants was 29 years, with a range of 20 - 35 years. The median age of participants was significantly higher in the insulin group as compared to the MNT group ( 30 vs. 27 years, $\mathrm{P}<0.001$ ). The median weight of the study participants was $58 \mathrm{~kg}$, with a range of $54-62 \mathrm{~kg}$. The median weight of the participants in the insulin group was signifi- 
Table 1. Maternal Baseline Characteristics

\begin{tabular}{|c|c|c|c|c|}
\hline \multirow{2}{*}{ Parameters } & \multirow{2}{*}{ Overall, median (IQR) } & \multicolumn{2}{|c|}{ Group } & \multirow{2}{*}{ P value } \\
\hline & & MNT $(n=121)$ & Insulin $(n=30)$ & \\
\hline Age (years)* & $29(25-30)$ & $27(25-30)$ & $30(29.3-32)$ & $<0.001^{\mathrm{a}}$ \\
\hline Weight $(\mathrm{kg})^{*}$ & $58(54-62)$ & $58(53-60)$ & $61(59.5-74.3)$ & $<0.001^{\mathrm{a}}$ \\
\hline Height $(\mathrm{m})^{*}$ & $1.56(1.52-1.6)$ & $1.56(1.51-1.58)$ & $1.58(1.55-1.61)$ & $0.014^{\mathrm{a}}$ \\
\hline Blood glucose (after 75-g OGTT) (mg/dL)* & $157(149-175)$ & $156(149-168)$ & $165.5(149.7-186.7)$ & $0.032^{\mathrm{a}}$ \\
\hline
\end{tabular}

Data are expressed as median (IQR), except primigravida expressed as frequency (percentage). *Statistically significant with P-value < 0.05 . aWilcoxon-Mann-Whitney U test. ${ }^{b}$ Chi-squared test. MNT: medical nutrition therapy; BMI: body mass index; 75-g OGTT: 75-gram oral glucose tolerance test; IQR: interquartile range.

cantly higher than the MNT group (61 vs. $58 \mathrm{~kg}, \mathrm{P}<0.001$ ). The participants in the insulin group also had a significantly higher median BMI than the MNT group (24.9 vs. $23.8 \mathrm{~kg} /$ $\left.\mathrm{m}^{2}, \mathrm{P}=0.003\right)$. In the insulin group, the median value of $75-\mathrm{g}$ OGTT was significantly higher than the MNT group (165.5 vs. $157 \mathrm{mg} / \mathrm{dL}, \mathrm{P}=0.032$ ).

The maternal outcomes in the two groups are depicted in Table 2. The development of gestational hypertension $(46.7 \%$ versus $18.2 \%$ ) and the rates of cesarean delivery (90.0\% versus $69.4 \%$ ) were significantly higher in the insulin-treated group than

Table 2. Maternal Outcomes

\begin{tabular}{|c|c|c|c|c|c|}
\hline \multirow{2}{*}{ Parameters } & \multirow{2}{*}{ Overall } & \multicolumn{2}{|c|}{ Group } & \multirow{2}{*}{$\operatorname{RR}(95 \% \mathrm{CI})$} & \multirow{2}{*}{ P value } \\
\hline & & MNT (n = 121) & Insulin $(\mathbf{n}=\mathbf{3 0})$ & & \\
\hline Gestational hypertension*,\# & $36(23.8 \%)$ & $22(18.2 \%)$ & $14(46.7 \%)$ & $0.71(0.52-0.89)$ & $0.001^{\mathrm{b}}$ \\
\hline $20-27^{6 / 7}$ & - & $2(9.1 \%)$ & $1(7.1 \%)$ & - & $1.000^{\mathrm{c}}$ \\
\hline$\geq 28$ & & $20(90.9 \%)$ & $13(92.9 \%)$ & & \\
\hline $\mathrm{PPH}^{\#}$ & $4(2.6 \%)$ & $4(3.3 \%)$ & $0(0.0 \%)$ & $1.26(0.64-1.38)$ & $0.585^{\mathrm{c}}$ \\
\hline Surgical site infection ${ }^{\#}$ & $5(3.3 \%)$ & $2(1.7 \%)$ & $3(10.0 \%)$ & $0.49(0.14-1.95)$ & $0.054^{\mathrm{c}}$ \\
\hline Indication for $\mathrm{CD}^{\#}$ & - & & & & $0.161^{\mathrm{c}}$ \\
\hline Previous CD & $26(23.4 \%)$ & $20(23.8 \%)$ & $6(22.2 \%)$ & & \\
\hline Dysfunctional labor & $9(8.1 \%)$ & $9(10.7 \%)$ & $0(0.0 \%)$ & & \\
\hline Obstructed labor & $8(7.2 \%)$ & $8(9.5 \%)$ & $0(0.0 \%)$ & & \\
\hline Malpresentation & $5(4.5 \%)$ & $4(4.8 \%)$ & $1(3.7 \%)$ & & \\
\hline $\mathrm{APH}$ & $2(1.8 \%)$ & $2(2.4 \%)$ & $0(0.0 \%)$ & & \\
\hline CPD & $2(1.8 \%)$ & $2(2.4 \%)$ & $0(0.0 \%)$ & & \\
\hline Contracted pelvis & $1(0 / 9 \%)$ & $1(1.2 \%)$ & $0(0.0 \%)$ & & \\
\hline Prolonged PROM & $1(0.9 \%)$ & $1(1.2 \%)$ & $0(0.0 \%)$ & & \\
\hline Uterine anomaly & $1(0.9 \%)$ & $0(0.0 \%)$ & $1(3.7 \%)$ & & \\
\hline
\end{tabular}

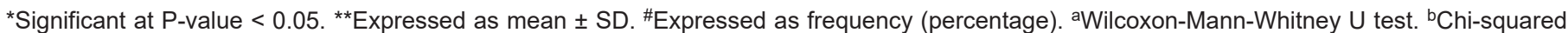
test. 'Fisher's exact test. MNT: medical nutrition therapy; RR: relative risk; $95 \%$ Cl: 95\% confidence interval; U: units; GA: gestational age; PPH: postpartum hemorrhage; CD: cesarean delivery; CDMR: cesarean done on maternal request; $\mathrm{APH}$ : antepartum hemorrhage; CPD: cephalopelvic disproportion; PROM: pre-labor rupture of membranes. 
Table 3. Neonatal Outcomes

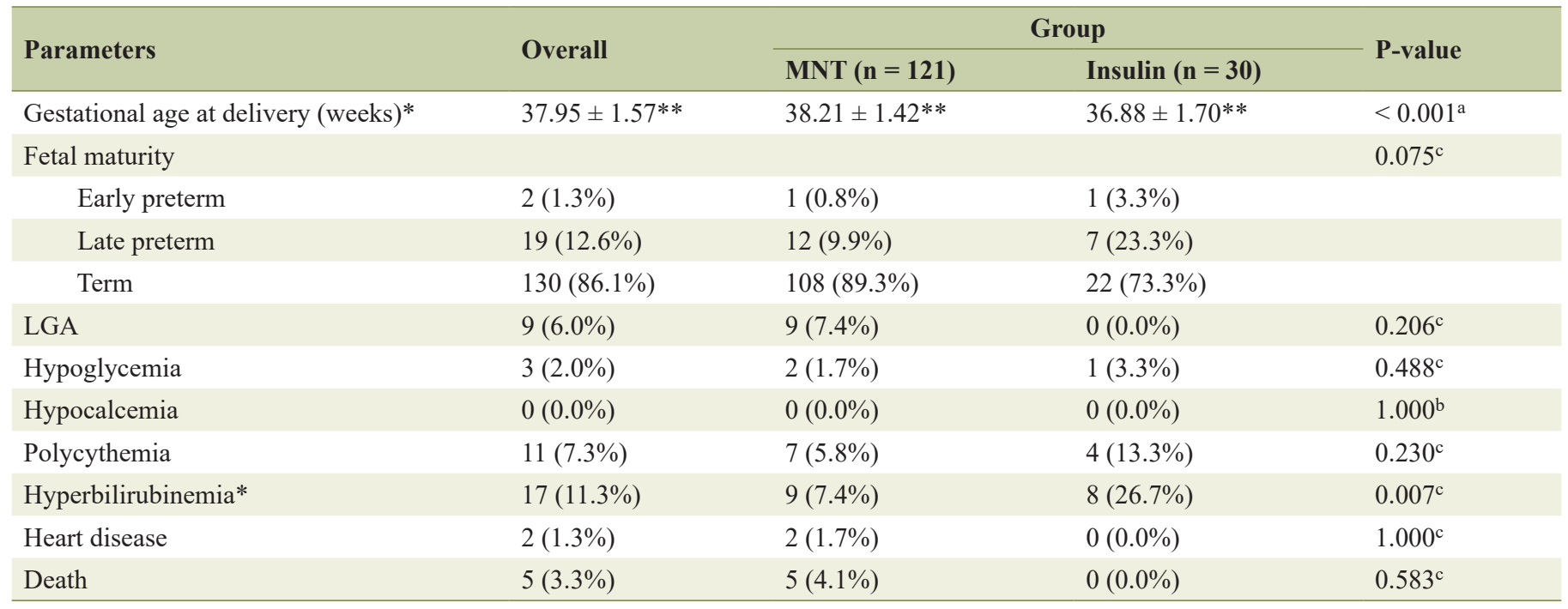

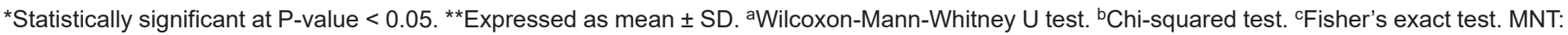
medical nutrition therapy; LGA: large for gestational age.

in the MNT-treated group. However, there was no difference in the gestational age at diagnosis of gestational hypertension, rates of instrumental delivery, postpartum hemorrhage, surgical site infection and the indications for cesarean delivery in the two groups.

Table 3 depicts the neonatal outcomes in the two study groups. The gestational age at delivery was earlier in the insulin-treated group (36.8 versus 38.2 weeks) than in the MNTtreated group. The overall rate of neonatal hyperbilirubinemia was $11.3 \%$, with the rate being significantly higher in the insulin-treated group (26.7\% versus $7.4 \%$ ) than in the MNT-treated group. The rates of fetal maturity at delivery (early preterm, late preterm and term neonates), LGA babies, hypoglycemia, hypocalcemia, polycythemia, neonatal congenital heart disease and neonatal deaths did not differ between the two groups.

The overall efficacy of MNT treatment was $80.1 \%$, with $19.9 \%$ of individuals requiring additional insulin therapy. Among those who required insulin, $83.3 \%$ achieved glycemic control, while euglycemia could not be achieved in the remaining $16.7 \%$.

ROC curve was plotted for predicting antenatal insulin requirement (Fig. 2). The cut-off which predicted need for antenatal insulin was gestational age $\geq 33.28$ weeks at diagnosis of GDM, maternal BMI $\geq 24.7 \mathrm{~kg} / \mathrm{m}^{2}$ and $75-\mathrm{g}$ OGTT value $\geq 162 \mathrm{mg} / \mathrm{dL}$. Of these, both maternal BMI $\geq 24.7 \mathrm{~kg} / \mathrm{m}^{2}$ and 75 -g OGTT value $\geq 162 \mathrm{mg} / \mathrm{dL}$ had the highest sensitivity of $63.3 \%$ in predicting antenatal insulin requirement (Table 4). Gestational age $\geq 33.28$ weeks at diagnosis of GDM had the highest specificity $(80.2 \%)$, while BMI $\geq 24.7 \mathrm{~kg} / \mathrm{m}^{2}$ had both, the highest positive predictive value (PPV) $(33.9 \%)$ as well as the highest negative predictive value (NPV) $(88.4 \%)$ for predicting antenatal insulin requirement.

\section{Discussion}

Our study shows that the median age, weight, BMI and value of 75-g OGTT at diagnosis of GDM were significantly higher in the insulin group as compared to the MNT group (30 vs. 27 years, 61 vs. $58 \mathrm{~kg}, 24.9$ vs. $23.8 \mathrm{~kg} / \mathrm{m}^{2}$ and 165.5 vs. 157 $\mathrm{mg} / \mathrm{dL}$ ). Previous studies have reported positive family history of diabetes mellitus, multiple abnormal values or single elevated 1-h value in OGTT and value of glycated hemoglobin (HbA1c) at diagnosis of GDM as independent predictors of antenatal insulin requirement [12-14]. In our study, following DIPSI protocols [11], we have done universal screening for GDM, used 75-g OGTT and tested for HbAlc only in those who were diagnosed with GDM in the first trimester.

\section{Predictors of antenatal insulin requirement}

In this study, we have attempted to identify the predictors of insulin requirement during pregnancy. Antenatal insulin was required in $19.9 \%$ cases in our study. Others have reported the requirement of insulin in women with GDM at around $10.0 \%$ [12].

Women with GDM requiring insulin had significantly higher BMI and a greater value of 75-g OGTT at diagnosis. The area under the ROC curve (AUROC) for predictive ability of 75-g OGTT value regarding the need for insulin was 0.627 (95\% confidence interval (CI): $0.507-0.747)$. The similar AUROC for BMI was found to be 0.673 (95\% CI: 0.569 - 0.777). Although statistically significant, both demonstrated poor diagnostic performance. AUROC was also calculated for gestational age at diagnosis, which showed insignificant results. We determined that the cut-off value which predicted need for antenatal insulin was gestational age $\geq 33.28$ weeks at diagnosis of GDM, maternal BMI $\geq$ $24.7 \mathrm{~kg} / \mathrm{m}^{2}$ and $75-\mathrm{g}$ OGTT value $\geq 162 \mathrm{mg} / \mathrm{dL}$. Therefore, this subset of women should be advised for strict follow-up and adherence to MNT and also should also be counselled 


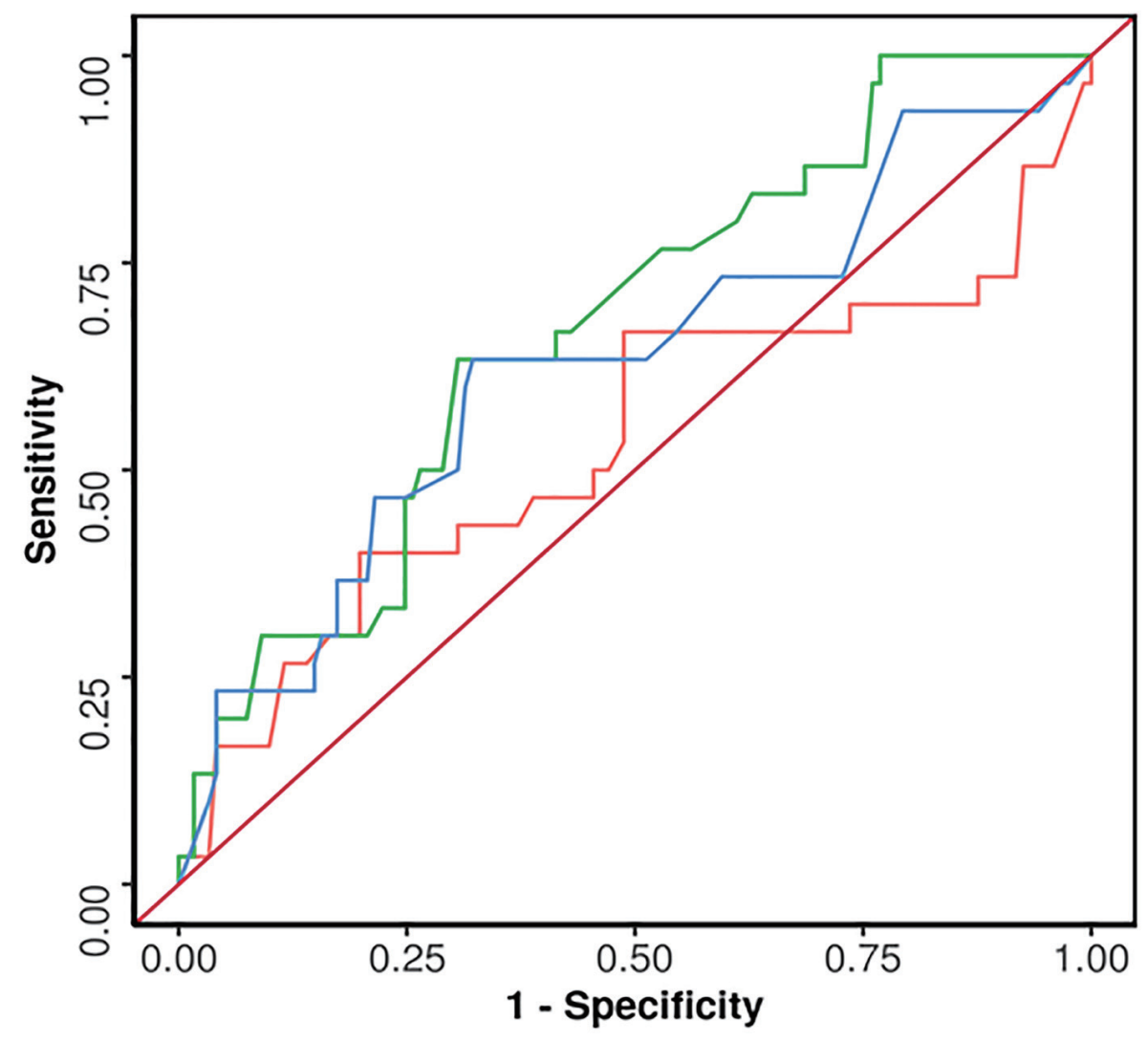

$\begin{array}{ll}\square & \text { Gestational Age at Diagnosis of GDM (Weeks) } \\ \text { BMI }(\mathrm{Kg} / \mathrm{m} 2) & \\ \text { Blood Glucose (After } 75 \mathrm{~g} \text { OGTT) }(\mathrm{mg} / \mathrm{dL})\end{array}$

Figure 2. ROC curve showing diagnostic performance of predictors for antenatal insulin requirement. ROC: receiver operating characteristic.

that they may go on to require insulin.

\section{Perinatal outcomes}

In our study, maternal and neonatal outcomes in women with
GDM were comparable across the two treatment groups of MNT and insulin except for rates of development of gestational hypertension (46.7\% versus $18.2 \%$ ), cesarean delivery $(90.0 \%$ versus $69.4 \%$ ) and neonatal hyperbilirubinemia (26.7\% versus $7.4 \%$ ), all of which were significantly higher in the insulintreated group than in the MNT group. This suggests that the

Table 4. Performance of Primary Diagnostic Parameters for Prediction of Antenatal Insulin Requirement

\begin{tabular}{llllll}
\hline Variable & Sensitivity & Specificity & PPV & NPV & $\begin{array}{l}\text { Diagnostic } \\
\text { accuracy }\end{array}$ \\
\hline $\begin{array}{l}\text { Gestational age at diagnosis of GDM } \\
\text { (weeks) (cutoff: } 33.286 \text { by ROC) }\end{array}$ & $30.0 \%(15-49)$ & $80.2 \%(72-87)$ & $27.3 \%(13-46)$ & $82.2 \%(74-89)$ & $70.2 \%(62-77)$ \\
BMI $\left(\mathrm{kg} / \mathrm{m}^{2}\right)$ (cutoff: 24.7 by ROC) & $63.3 \%(44-80)$ & $69.4 \%(60-77)$ & $33.9 \%(22-48)$ & $88.4 \%(80-94)$ & $68.2 \%(60-76)$ \\
BMI $\left(\mathrm{kg} / \mathrm{m}^{2}\right)$ (cutoff: $\left.\geq 25\right)$ & $50.0 \%(31-69)$ & $73.6 \%(65-81)$ & $31.9 \%(19-47)$ & $85.6 \%(77-92)$ & $68.9 \%(61-76)$ \\
$\begin{array}{l}\text { Blood glucose (after } 75 \text {-g OGTT) } \\
(\mathrm{mg} / \mathrm{dL})(\text { cutoff: } 162 \text { by ROC) }\end{array}$ & $63.3 \%(44-80)$ & $67.8 \%(59-76)$ & $32.8 \%(21-46)$ & $88.2 \%(80-94)$ & $66.9 \%(59-74)$ \\
\hline
\end{tabular}

PPV: positive predictive value; NPV: negative predictive value; GDM: gestational diabetes mellitus; BMI: body mass index; 75-g OGTT: 75-gram oral glucose tolerance test; ROC: receiver operating characteristic. 
addition of insulin is not protective for the development of adverse perinatal outcomes. Mothers receiving insulin should be counselled regarding greater risk of hyperbilirubinemia in her newborn, which might require phototherapy, admission to neonatal intensive care unit (NICU) and prolonged hospital stay. There were no stillbirths in any of the groups.

In a review by Mastrogiannis et al, the relationship between GDM and gestational hypertension was ascertained [14]. This relationship was hypothesized to be due to insulin resistance. It was also stated that this risk was far greater in obese women and in the third trimester. In our study, the higher incidence of gestational hypertension in the insulin group can be partly explained by the significant proportion of obese individuals in the same group. Other researchers have reported either no difference in maternal and neonatal complications in GDM women with and without insulin therapy [15], or have found nulliparity and cesarean delivery to be associated with neonatal morbidity in women with GDM [16]. A recent meta-analysis found that insulin use had a significant increase in the risk of preeclampsia, NICU admission, neonatal hypoglycemia and macrosomia compared to metformin use antenatally [17]. In our study, we did not use either metformin or glyburide for glycemic control.

The three commonest indications of cesarean delivery in our study were women with previous cesarean delivery not willing for trial of labor, non-reassuring fetal heart status and failed induction. A Cochrane review also supports higher cesarean rates with the use of continuous fetal monitoring [18]. In addition to this, there were a significantly higher number of primigravida in the insulin group $(70.0 \%$ versus $43.0 \%)$ who either refused for induction of labor or had failed induction contributing to $37 \%$ cases. Moreover, it was seen that the mean gestational age in the insulin-treated group was 36 weeks, thus increasing the chances of failed induction.

\section{Strengths of the study}

Ours was a prospective study, unlike previous studies, many of which were retrospective in nature. Due to this, missing data for maternal and neonatal outcomes were minimized. Also, we have followed the recommended DIPSI criteria for diagnosis of GDM, which is single step and is more feasible to perform in a developing country like ours. Apart from this, parameters predictive for initiation of insulin requirement were also studied prospectively.

\section{Limitations of the study}

The sample size was limited in our study due to the disruptions in services caused by the COVID-19 pandemic. Also, we monitored the compliance to MNT as per the words of the participants, with no home visits. We have not considered the type of insulin used by women in this study.

\section{Future research}

Future research should focus on establishing national registry of women with GDM, so that more data are available for predicting the requirement of insulin, as well as quantifying the adverse perinatal outcomes.

To conclude, this prospective observational cohort study was conducted on 151 women with GDM with the primary objective of identifying parameters that predict antenatal insulin requirement. The secondary objective was to compare the perinatal outcomes in MNT-treated versus insulin-treated groups. Our study showed that gestational age $\geq 33.28$ weeks at diagnosis of GDM, maternal BMI $\geq 24.7 \mathrm{~kg} / \mathrm{m}^{2}$ and $75-\mathrm{g}$ OGTT value $\geq 162 \mathrm{mg} / \mathrm{dL}$ were predictors of insulin requirement in pregnancy. The median age, weight, BMI, value of 75-g OGTT at diagnosis of GDM, rate of cesarean deliveries, gestational hypertension and neonatal hyperbilirubinemia were significantly higher in the insulin-treated group than in the MNT group.

\section{Acknowledgments}

None to declare.

\section{Financial Disclosure}

None to declare.

\section{Conflict of Interest}

None to declare.

\section{Informed Consent}

Written informed consent was obtained from the study participants at the time of enrolment.

\section{Author Contributions}

Neelu Rajkumar Sontakke helped with data collection, compiled the data and wrote the first draft of the manuscript. Sweta Singh helped with patient recruitment, data collection and compilation and revised the manuscript. Subarna Mitra conceived the study and helped with patient recruitment and data collection. Kishore Kumar Behera helped with data collection and gave critical endocrine inputs. Pankaj Kumar Mohanty helped with data collection and gave critical neonatal inputs. All authors have read and agree to the final version to be published.

\section{Data Availability}

The authors declare that data supporting the findings of this study are available within the article. 


\section{References}

1. ACOG Practice Bulletin No. 190: gestational diabetes mellitus. Obstet Gynecol. 2018;131(2):e49-e64.

2. International Diabetes Federation. IDF Diabetes Atlas, 9th edn. Brussels, Belgium: International Diabetes Federation, 2019.

3. Caughey AB, Cheng YW, Stotland NE, Washington AE, Escobar GJ. Maternal and paternal race/ethnicity are both associated with gestational diabetes. Am J Obstet Gynecol. 2010;202(6):616 e611-615.

4. Lee KW, Ching SM, Ramachandran V, Yee A, Hoo FK, Chia YC, Wan Sulaiman WA, et al. Prevalence and risk factors of gestational diabetes mellitus in Asia: a systematic review and meta-analysis. BMC Pregnancy Childbirth. 2018;18(1):494.

5. Khan S, Bal H, Khan ID, Paul D. Prevalence of gestational diabetes mellitus in an urban Indian cohort using diabetes in pregnancy study group in India (DIPSI) criteria - validating one-step approach. International Journal of Medicine and Medical Research. 2019;4(2):13-19.

6. Li MF, Ma L, Yu TP, Zhu Y, Chen MY, Liu Y, Li LX. Adverse maternal and neonatal outcomes in pregnant women with abnormal glucose metabolism. Diabetes Res Clin Pract. 2020;161:108085.

7. Coustan DR, Lowe LP, Metzger BE, Dyer AR, International Association of D, Pregnancy Study G. The Hyperglycemia and Adverse Pregnancy Outcome (HAPO) study: paving the way for new diagnostic criteria for gestational diabetes mellitus. Am J Obstet Gynecol. 2010;202(6):654 e651-656.

8. HAPO Study Cooperative Research Group, Metzger BE, Lowe LP, Dyer AR, Trimble ER, Chaovarindr U, Coustan DR, et al. Hyperglycemia and adverse pregnancy outcomes. N Engl J Med. 2008;358(19):1991-2002.

9. Hartling L, Dryden DM, Guthrie A, Muise M, Vandermeer B, Donovan L. Benefits and harms of treating gestational diabetes mellitus: a systematic review and metaanalysis for the U.S. Preventive Services Task Force and the National Institutes of Health Office of Medical Appli- cations of Research. Ann Intern Med. 2013;159(2):123129.

10. Horvath K, Koch K, Jeitler K, Matyas E, Bender R, Bastian $\mathrm{H}$, Lange $\mathrm{S}$, et al. Effects of treatment in women with gestational diabetes mellitus: systematic review and meta-analysis. BMJ. 2010;340:c1395.

11. Seshiah V, Das AK, Balaji V, Joshi SR, Parikh MN, Gupta S, Diabetes in Pregnancy Study G. Gestational diabetes mellitus - guidelines. J Assoc Physicians India. 2006;54:622-628.

12. Mitra S, Nayak PK, Sahoo J, Mathew A, Padma A, Kamalanathan S, Agrawal S. Predictors for antenatal insulin requirement in gestational diabetes. Gynecol Endocrinol. 2014;30(8):565-568.

13. Ducarme G, Desroys du Roure F, Grange J, Vital M, Le Thuaut A, Crespin-Delcourt I. Predictive factors of subsequent insulin requirement for glycemic control during pregnancy at diagnosis of gestational diabetes mellitus. Int J Gynaecol Obstet. 2019;144(3):265-270.

14. Mastrogiannis DS, Spiliopoulos M, Mulla W, Homko CJ. Insulin resistance: the possible link between gestational diabetes mellitus and hypertensive disorders of pregnancy. Curr Diab Rep. 2009;9(4):296-302.

15. Watanabe M, Katayama A, Kagawa H, Ogawa D, Wada J. Risk factors for the requirement of antenatal insulin treatment in gestational diabetes mellitus. J Diabetes Res. 2016;2016:9648798.

16. Ducarme G, Desroys Du Roure F, Le Thuaut A, Grange J, Dimet J, Crepin-Delcourt I. Efficacy of maternal and biological parameters at the time of diagnosis of gestational diabetes mellitus in predicting neonatal morbidity. Eur J Obstet Gynecol Reprod Biol. 2018;221:113-118.

17. Guo L, Ma J, Tang J, Hu D, Zhang W, Zhao X. Comparative efficacy and safety of metformin, glyburide, and insulin in treating gestational diabetes mellitus: a metaanalysis. J Diabetes Res. 2019;2019:9804708.

18. Alfirevic Z, Devane D, Gyte GM, Cuthbert A. Continuous cardiotocography (CTG) as a form of electronic fetal monitoring (EFM) for fetal assessment during labour. Cochrane Database Syst Rev. 2017;2:CD006066. 